Revista Iberoamericana, Vol. LXXVII, Núm. 235, Abril-Junio 2011, 299-311

\title{
CRONOLOGÍA DE NANCY MOREJÓN
}

\author{
POR \\ JuANAmaría CORDONES-COOK \\ University of Missouri
}

1944 Nació el 7 de agosto en el hospital Maternidad Obrera, en Marianao, La Habana (Cuba). Creció en el barrio Los Sitios en Centro Habana. Entre 1950 y 1956 realizó estudios de primaria en el Colegio Academia Laplace, también de Centro Habana. En 1957 matriculó estudios de Segunda Enseñanza en el Instituto de La Habana.

1961 Se graduó de Bachiller en Letras en el Instituto de La Habana.

1962 Inició una Licenciatura en Lengua y Literatura Francesa en la Universidad de La Habana.

Primer poema publicado en la antología Novísima poesía cubana, de Ana María Simo y Reynaldo García Ramos (La Habana: El Puente).

Se publicó Mutismos (poemario) (La Habana: El Puente).

1964 Se publicó Amor, ciudad atribuida (poemario)(La Habana: El Puente).

El poema “Los heraldos negros" recibió el Premio de Poesía Rubén Martínez Villena de la Escuela de Artes y Letras de la Universidad de La Habana.

1966 Terminó la licenciatura en Lengua y Literatura Francesa, Suma Cum Laude, en la Universidad de La Habana. Tesis de grado sobre la obra del martiniqueño Aimé Césaire, de quien tradujo Una temporada en el Congo para ser puesta en escena por el director Roberto Blanco. [Su trabajo como traductora del francés al español se ha seguido desarrollando con obras de Paul Éluard, Arthur Rimbaud, Jacques Roumain, Édouard Glissant y Ernest Pépin, entre otros.] 
Primera mención por Richard trajo su flauta y otros argumentos en el Premio de Poesía Julian del Casal de la UNEAC, 1966

1967 Se publicó Richard trajo su flauta y otros argumentos (poemario) (La Habana: Unión, Colección Cuadernos). Mención de la Unión Nacional de Escritores y Artistas de Cuba (UNEAC) por un jurado compuesto por José Lezama Lima, Nicolás Guillén, Regino Pedroso, José Agustín Goytisolo, Roque Dalton, Oscar Oliva, Jaime Augusto Shelley y Yannis Ritsos.

Formó parte del Encuentro por el Centenario de Rubén Darío que convocara la Casa de las Américas en Varadero (Cuba) al que asistieron los poetas más destacados de la época: Carlos Pellicer (México); Mario Benedetti (Uruguay); Enrique Lihn (Chile); Thiago de Mello (Brasil); René Depestre (Haití); César Calvo (Perú); Francisco Urondo (Argentina); Roque Dalton (El Salvador); César Fernández Moreno (Argentina); Ida Vitale (Uruguay); Heberto Padilla; Roberto Fernández Retamar; Pablo Armando Fernández; y Miguel Barnet (Cuba), entre otros.

1971 Se publicó Lengua de pájaro, en colaboración con Carmen Gonce (monografía etno-histórica sobre Nicaro) (La Habana: Ciencias Sociales).

1972 Editó Recopilación de textos sobre Nicolás Guillén (La Habana: Casa de las Américas, Serie Valoración Múltiple).

1975 Se publicó "Mujer negra" en la revista Casa de las Américas, en un número especial dedicado al Año Internacional de la Mujer.

1979 Se publicó Parajes de una época (poemario) (La Habana: Letras Cubanas, Colección Mínima).

Se publicó la traducción de Las armas cotidianas, de Paul Laraque (La Habana: Casa de las Américas, Colección Premio).

Asistió al Festival Internacional de Poesía Pushkin, en Moscú. Participó en el congreso "Rompiendo Barreras", organizado por la Universidad Interamericana de Puerto Rico, en San Juan.

1980 Se publicó Poemas (antología). Selección y prólogo de Efraín Huerta. Ilustración de cubierta de Wifredo Lam (México: UNAM).

Revista Iberoamericana, Vol. LXXVII, Núm. 235, Abril-Junio 2011, 299-311 
Primera mención por Elogio de la danza, en el Concurso de Poesía del Taller Coreográfico de Gloria Contreras auspiciado por la UNAM, 1980.

1982 Se publicó Elogio de la danza (poemario) (México: UNAM, Colección Cuadernos de Poesía).

Se publicó Octubre imprescindible (poemario) (La Habana: Unión).

Tradujo versiones de las piezas teatrales La duodécima noche, de William Shakespeare, y El burgués gentilhombre, de Molière, para puestas en escena del cubano Vicente Revuelta y la uruguaya Sara Larocca, respectivamente, en el Festival Internacional de Teatro de La Habana.

Se publicó Nación y mestizaje en Nicolás Guillén (La Habana: Unión). Premio Nacional de Ensayo Enrique José Varona de la UNEAC.

1983 Premio Mirta Aguirre por el libro Nación y mestizaje en Nicolás Guillén.

Primera visita a Estados Unidos. Ofreció un recital en el Poetry Room de la Universidad de Harvard, el primero de numerosos recitales que ofreciera habitualmente en el mundo académico de los Estados Unidos hasta el 2003.

1984 Sepublicó Grenada Notebook/Cuadernode Granada(poemario). Traducción de Lisa Davis (Nueva York: Círculo de Cultura Cubana). La edición Príncipe fue publicada por Casa de las Américas.

Ofreció recital de poesía en el St. Catherine's College de la Universidad de Oxford (Inglaterra).

1985 Se publicó Where the Island Sleeps Like a Wing (primera antología bilingüe). Traducción de Kathleen Weaver (San Francisco: The Black Scholar Press), antología seleccionada entre los diez mejores libros de poesía del año por el diario The San Francisco Chronicle, de California.

Se publicó Poems (antología). Selección de Sandra Levinson (Nueva York: Center for Cuban Studies) (Libro y audio-cassette).

1986 Se publicó Piedra pulida (poemario) (La Habana: Letras Cubanas). Premio de la Crítica.

Revista Iberoamericana, Vol. LXXVII, Núm. 235, Abril-Junio 2011, 299-311 
Es nombrada Directora del Centro de Estudios del Caribe de la Casa de las Américas, donde trabajara hasta 1993.

1987 Noviembre 18, falleció su padre, Felipe Morejón Noyola.

1988 Se publicó Fundación de la imagen: Ensayo (La Habana: Letras Cubanas).

Participó en el Festival Mundial de Poetas Latinos en la Ciudad de México.

1989 Se publicó Dos poemas de Nancy Morejón, plaquette con dibujos y diseño de Rolando Estévez (Matanzas, Cuba: Vigía).

Invitada de Honor al 54 Congreso Internacional del Pen Club Internacional de Toronto y Montreal.

Distinción 23 de Agosto que otorga el Secretariado de la Fundación de Mujeres Cubanas.

1990 Se publicó Ours the Earth (antología). Traducción de Joseph Pereira (Mona: Instituto del Caribe de la University of the West Indies).

1991 Tradujo Remolino de palabras libres, de Ernest Pépin (La Habana: Casa de las Américas).

Se publicó Baladas para un sueño (poemario) (La Habana: Unión, Colección Ciclos).

Se publicó la traducción de Remolino de palabras libres, de Ernest Pépin (La Habana: Casa de las Américas, Colección Premio).

Medalla 30 Años de Alfabetización.

Participó, con la compositora Marta Valdés, en el Festival Internacional de Poesía de Trois Rivières (Québec, Canadá) y en el coloquio "Dos poesías: una lengua", durante los Cursos de Verano de la Universidad Complutense de Madrid (España).

1992 Invitada al Congreso Nacional de Escritores Sudafricanos (COSAW), visitó la República Sudafricana ofreciendo recitales y talleres literarios. Invitada de

Revista Iberoamericana, Vol. LXXVII, Núm. 235, Abril-Junio 2011, 299-311 
honor a la III Conferencia de Escritores del Caribe, celebrado en Curazao; así como a la V Edición de CARIFESTA en Trinidad y Tobago.

Primera mención por Paisaje célebre en el Premio Internacional de Poesía "Antonio Pérez Bonalde" de Venezuela.

1993 Se publicó Paisaje célebre (Caracas: Fundarte).

Nombrada Directora de la Editorial PM de la Fundación Pablo Milanés hasta 1995.

Se publicó Poemas de amor y de muerte (antología) (Toulouse: Revista Caravelle).

1994 Se publicó Le Chaînon Poétique (antología bilingüe). Traducción del francés de Sandra Monet-Descombey. Ilustración de cubierta del pintor dominicano José Castillo. Prefacio de Delia Blanco y Pilar Paliès (París: Champignysur-Marne, Edition L.C.J.).

1995 Designada asesora de la Dirección del Teatro Nacional de Cuba que dirigió Nisia Agüero hasta el 2000.

Abril 28-29 Simposio Internacional en Honor de Nancy Morejón, organizado por Juanamaría Cordones-Cook, Universidad de Missouri, Columbia.

1996 Se publicó El río de Martín Pérez y otros poemas (antología). Plaquette con dibujos y diseño de Rolando Estévez (Matanzas, Cuba: Vigía).

Se publicó Botella al mar (antología). Selección y prólogo de Adolfo Ayuso (Zaragoza: Olifante).

Se publicó su traducción de Arc en ciel, l'espoir (Arco iris, la esperanza) de Nicole Cage Florentiny (La Habana: Casa de las Américas, Colección Premio).

Lectura comentada de su poesía, "Apariencia y razón de una poética", en el marco del Encuentro de Poetas Cubanos y Catalanes como homenaje al poeta Rafael Alberti, auspiciado por la Fundación que lleva su nombre y realizada en su sede del Puerto de Santa María, Bahía de Cádiz, en España.

Revista Iberoamericana, Vol. LXXVII, Núm. 235, Abril-Junio 2011, 299-311 
Se publicó el número especial de Afro-Hispanic Review (primavera 1996) dedicado a la obra de Nancy Morejón. (Ponencias e intervenciones del Simposio Internacional en Honor a la obra de Morejón en la Universidad de Missouri, en abril de 1995).

1997 Se publicó Elogio y paisaje, compilación de Elogio de la danza y Paisaje célebre a cargo de Gerardo Fulleda León (La Habana: Unión, Colección La Rueda Dentada). Premio de la Crítica.

Febrero 15, murió su madre Angélica Hernández Domínguez.

1998 Se publicó Divertimento y otros poemas (antología) (Madrid-La Habana: Sureditors, Colección Milhojas).

Se publicó Fastos y otros poemas, su traducción de Fastes: Poemes de Édouard Glissant. Plaquette con dibujos y diseño de Rolando Estévez (Matanzas: Vigía).

1999 Ingresó como Miembro de Número en la Academia Cubana de la Lengua.

Se publicó Richard trajo su flauta y otros poemas (antología). Selección y prólogo de Mario Benedetti (Madrid:Visor).

Se publicó Singular Like a Bird, una recopilación de estudios críticos sobre su obra que realizara Miriam De Costa-Willis (Washington, DC: Howard University Press).

Conversación de Nancy Morejón con Juanamaría Cordones-Cook (Universidad de Missouri, Columbia). (Video presentado en el Festival Internacional del Nuevo Cine Latinoamericano en diciembre, La Habana)

2000 Se publicó La Quinta de Los Molinos (poesía) (La Habana: Letras Cubanas). Premio de la Crítica.

Designada directora del Centro de Estudios del Caribe de la Casa de las Américas hasta el 2006.

En la galería René Portocarrero del Teatro Nacional se inaugura Pasatiempos, su primera exposición de dibujos, que presentan los poetas Aitana Alberti y Pablo Armando Fernández.

Revista Iberoamericana, Vol. LXXVII, Núm. 235, Abril-Junio 2011, 299-311 
$2001 \quad$ Premio Nacional de Literatura.

Se publicó Rumreiche Landschaft. Versión alemana de Paisaje célebre. Traducción de Ineke Phaf-Reinberger (Berlín: Colega Verlag).

Se publicó Black Woman and other Poems (antología bilingüe). Traducción e introducción de Jean Andrews (Londres: Mango).

La Biblioteca Nacional José Martí la acreditó como Lectora Honoraria de esa institución por haber recibido el Premio Nacional de Literatura 2001.

2002 Se publicó Cántico de la huella (poema). Plaquette con dibujos y diseño de Rolando Estévez (Matanzas: Vigía, Colección Inicios).

Se publicó Cuerda veloz (Antología de poemas 1962-1992). Prólogo y selección de la autora (La Habana: Letras Cubanas, Colección Premio Nacional de Literatura).

Distinción Gitana Tropical. Otorgada, en el Teatro Nacional de Cuba el 2 de marzo por la Dirección Provincial de Cultura de Ciudad de La Habana.

Se publicó la segunda edición de La Quinta de los Molinos. Ilustración de cubierta: óleo de Amelia Peláez de título homónimo. Ilustraciones interiores de Reynaldo González (La Habana: Letras Cubanas).

Se publicó la segunda edición de Lengua de pájaro, en colaboración con Carmen Gonce (monografía etno-histórica sobre Nicaro) (Santiago de Cuba: Oriente).

Distinción Medalla José María Heredia y Heredia. Otorgada en la Casa de Heredia por la Dirección Provincial de Cultura de Santiago de Cuba el 9 de marzo.

Se publicó una nueva edición de Fastos y otros poemas, su traducción de Fastes: poemes de Édouard Glissant (La Habana: Casa de las Américas, Colección Pasamanos).

Primera exposición de dibujos, Pasatiempos, que presentara Pablo Armando Fernández en la Galería René Portocarrero, del Teatro Nacional de Cuba. Curadora: Orquídea García. Palabras del catálogo de Aitana Alberti.

Revista Iberoamericana, Vol. LXXVII, Núm.235, Abril-Junio 2011, 299-311 
2003 Se publicó Looking Within / Mirar adentro: Selected Poems. Poemas escogidos. 1954-2000 (antología bilingüe) (Detroit: Wayne State University Press, African American Life Series). Edición, anotación e introducción de Juanamaría Cordones-Cook. Traducciones de Gabriel Abudu, David Frye, Nancy Abraham Hall, Mirta Quintanales, Heather Rosario Sievert y Kathleen Weaver (best seller de Wayne State University Press). Esta antología fue presentada en el marco de un coloquio en la Conferencia Internacional de LASA, el 27 de marzo (Dallas, Texas).

Se publicó Där ön sover som en vinge. Traducción al sueco hecha por Lasse Sóderberg (Malmo, Suecia: Aura Latina).

Recibió la Réplica del Machete de Máximo Gómez que le otorgara el Ministro de las Fuerzas Armadas de Cuba, el 16 de abril.

2004 Se publicó Ana Mendieta (poema, versión biligüe), plaquette con dibujos y diseño de Rolando Estévez (Matanzas: Vigía, Colección del Estero).

Se publicó With Eyes and Soul: Images of Cuba (antología bilingüe). Fotografías de Milton Rogovin. Traducción de Pamela Carmell y David Frye (Buffalo: White Pine Press).

Se publicó Poética de los altares (ensayos) (La Habana: Letras Cubanas, Colección Mínima).

El 12 de febrero fue condecorada con las Insignias de Oficial de la Orden al Mérito de la República de Francia por el conjunto de su obra literaria.

Se publicó El dulce abismo (cartas, dibujos, apuntes de los familiares de los Cinco Héroes cubanos prisioneros en EE.UU.). Presentación de Alice Walker. Prólogo de Nancy Morejón (La Habana: José Martí).

Segunda exposición de dibujos, Pensamientos en La Habana, que presentara el poeta Sigfredo Ariel en la Galería "Raúl Martínez", en el Palacio del Segundo Cabo, sede del Instituto Cubano del Libro situado frente a la Plaza de Armas de La Habana Vieja (noviembre).

El 20 de julio recibió el Diploma Honrar, honra que le otorgara la Sociedad Cultural José Martí por los méritos de su obra.

Revista Iberoamericana, Vol. LXXVII, Núm. 235, Abril-Junio 2011, 299-311 
Por el conjunto de su obra recibió el Premio Yari-Yari, de la Universidad de Nueva York (NYU).

2005 Se publicó Carbones silvestres (poemario). Ilustración de cubierta de Eduardo Roca (Choco) (La Habana: Letras Cubanas).

Se publicó Ensayos. Selección y prólogo de Trinidad Pérez Valdés (La Habana: Letras Cubanas)

Se publicó Estos otros argumentos (Antología de poemas 1967-2005). Introducción y selección de Teresa Melo. Prólogo de Marino Wilson Jay. Ilustraciones de Jorge Knight Vera (Santiago de Cuba: Oriente).

Se publicó Entre leopardos (antología). Prólogo de Carilda Oliver Labra. Dibujos de la autora (Matanzas: Manglar y Uvero, Colección Homenaje). Se publicó España en Nicolás Guillén. Discurso de ingreso a la Academia Cubana de la Lengua. Dibujo de cubierta de la autora (La Habana: Unión, Colección Vagabundo del Alba).

Se publicó el número especial dedicado a Nancy Morejón de Callaloo: A Journal of African Diaspora Arts and Letters (Volumen 28, Número 4). Edición a cargo de Juanamaría Cordones-Cook.

Participó en un congreso de LART (Latin Artists Round Table de la ciudad de Nueva York) que auspiciara la Universidad de Santo Domingo (República Dominicana).

El 9 de agosto de 2005 recibió la Orden Majadahonda en la Unión de Escritores y Artistas de Cuba por la proyección internacional de su obra.

Conferencia magistral en el Congreso Internacional de Letras Femeninas, en octubre, Tegucigalpa (Honduras).

2006 La XV Feria Internacional del Libro de Cuba fue dedicada a Nancy Morejón y Ángel Augier en el transcurso de la cual, a lo largo de la Isla, recibió innumerables premios y distinciones.

Es designada Asesora de Casa de las Américas.

Revista Iberoamericana, Vol. LXXVII, Núm. 235, Abril-Junio 2011, 299-311
ISSN 2154-4794 (Electrónico) 
Se publicó Pierrot y la luna (Poema dramático). Diseño, dibujos y caligrafía de Rolando Estévez. Estudio preliminar de Juanamaría Cordones-Cook (Matanzas: Vigía).

Se publicó Antología poética (1962-2000). Selección y prólogo de Gerardo Fulleda León (Caracas: Monte Ávila).

Febrero 8: "Coloquio en honor de Nancy Morejón", organizado por la UNEAC, en La Habana. Presidido por Gerardo Fulleda León y con la participación de Miguel Barnet, Juanamaría Cordones-Cook, Pablo Armando Fernández, Teresa Melo e Ineke Phaf-Rheinberger.

Se publicó Dosestudios sobre Nicolás Guillén (Camagüey: Ácana, Colección Suma y Reflejo).

Se publicó Pluma al viento: Prosa periodística (Santiago de Cuba: Oriente).

Febrero 13: Coloquio "La palabra como manto". Homenaje a Nancy Morejón, organizado por Ediciones Vigía, que tuviera lugar en la sede de la Asociación Cubana de Artesanos Artistas, Matanzas. Participantes: Carilda Oliver Labra, Georgina Herrera, Juanamaría Cordones-Cook, Gerardo Fulleda León, Sigfredo Ariel.

Febrero 13: Cuarta exposición de dibujos en Matanzas en ocasión de la inauguración de la XV Feria Internacional del Libro en Matanzas.

Marzo 21: Día Mundial de la Poesía, fue anunciado, en la sede de la UNESCO de París, el Premio de Poesía Corona de Oro que le sería otorgado el mes de agosto en la XLV edición del Festival Internacional de Poesía de Struga, Macedonia.

Recibió el Premio de Poesía Corona de Oro, el domingo 27 de agosto, sobre el puente que cruza el río Drim, durante la XLV edición del Festival Noches de Poesía de Struga, Macedonia.

Se publicó Poemas selectos. Antología trilingüe. "Palabras para aceptar la Corona de Oro", de Nancy Morejón. Introducción de Mairym Cruz-Bernal. Traducción al macedonio de Ognena Nikuljska (español) y Zoran Ančevski (inglés) (Struga: Golden Wreath of Struga Poetry Evenings, 2006). Foto de Liborio Noval.

Revista Iberoamericana, Vol. LXXVII, Núm. 235, Abril-Junio 2011, 299-311 
Durante el mes de septiembre fue invitada por diversas instituciones culturales para ofrecer recitales en varias ciudades de Bulgaria. También visitó la Unión de Escritores de Atenas, Grecia.

28 de septiembre: Muestra de dibujos de los Pierrots de Morejón como tributo a García Lorca a los 70 años de su ejecución, en la Galería Espacio Abierto de la revista Revolución y Cultura, La Habana.

En noviembre, participó en Caracas en la II Feria Internacional del Libro de Venezuela. En ese marco se produjo el lanzamiento de la Antología poética que fuera publicada por la editorial Monte Ávila.

Pasó a integrar el Jurado Permanente del Premio Carbet presidido por Édouard Glissant que tuviera lugar en la ciudad de Pointe-à-Pitre, capital de la Guadalupe, Antillas francesas.

2007 Recibió el Premio de Poesía Rafael Alberti auspiciado por Andalucía en el XII Festival Internacional de Poesía de La Habana que presidiera Rogelio MartínezFuré, el martes 29 de mayo, a las seis de la tarde. La velada inaugural del evento fue efectuada en la Basílica Menor del Convento de San Francisco de Asís en La Habana Vieja. El jurado, presidido por el poeta Pablo Armando Fernández, Premio Nacional de Literatura, estuvo integrado además por Aitana Alberti y por Manuel José Vallejo, quien preside la Sociedad de Beneficencia de los Naturales de Andalucía y sus Descendientes. Leída el acta, donde expresa el jurado que este premio se otorga "por la relevancia de la totalidad de la obra" de la poeta, el presidente del jurado le hizo entrega a la premiada de una hermosa serigrafía de Rafael Alberti que data de 1973.

El 27 de abril, en el marco de la celebración del I Festival Internacional de Poesía de Santo Domingo, fue declarada Visitante Distinguida de la capital de la República Dominicana a solicitud del Alcalde Sr. Roberto Salcedo. La ceremonia fue efectuada a las nueve de la mañana en la sede del Ayuntamiento del Distrito Nacional enclavada en la zona colonial.

La "Nancy Morejón Special Collection of Afro-Romance Literature and Culture", dedicada en honor de la poeta a la literatura y la cultura de la diáspora africana en América Latina, es fundada por Juanamaría Cordones-Cook en el Museo de Antropología de la Universidad de Missouri, Columbia.

Revista Iberoamericana, Vol. LXXVII, Núm. 235, Abril-Junio 2011, 299-311
ISSN 2154-4794 (Electrónico) 
2008 En abril, fue elegida Presidenta de la Asociación de Escritores de la UNEAC.

En mayo, recibió el premio internacional Escritora Galega Universal entregado por la Asociación de Escritores en Lingua Galega (AELG) por el conjunto de su obra en la Cea das letras galegas, en Santiago de Compostela, España. Se publicará en Galicia una antología de sus poemas traducida al gallego.

En el mes de octubre, la Universidad de Salamancapublicó El huerto magnífico de todos, junto con una antología de su obra prologada y seleccionada por el poeta peruano Alfredo Pérez Alencart. Esa ciudad la condecoró con la condición de Visitante Ilustre en el marco del XI Encuentro de Poetas Iberoamericanos que le rindiera homenaje a la poeta.

Obtiene el Premio de Honor CUBADISCO 2008.

Se publicó la traducción de Cascadas, de Édouard Glissant (Matanzas, Vigía).

2009 El 27 de enero, la Universidad de Cergy-Pontoise le concedió el doctorado Honoris Causa.

La Oficina Regional de la UNESCO le entregó la Medalla Víctor Hugo. Participó en la Feria del Libro de Estambul, Turquía.

En diciembre, aparece Soltando amarras y memorias: Mundo y poesía de nancy Morejón, estudio monográfico de Juanamaría Cordones-Cook (Santiago de Chile: Cuarto Propio, colección Ensayo/Literatura).

2010 Tiene una destacada participación en el Festival Internacional de Poesía Split This Rock de Washington, DC. Además de ofrecer recitales en la Conferencia Mundial de Asuntos Exteriores de la Universidad de Nebraska, también las imparte en la Universidad de York. Invitada a Nueva York por el Grupo LART.

Aparece el nuevo poemario Peñalver 51 en el catálogo de la Fundación Sinsonte de Zamora, España, así como la breve antología Persona, complilada por Gerardo Fulleda León en la Colección Sur de La Habana, como resultado de haber recibido el Premio Rafael Alberti en 2007. Estos dos títulos fueron presentados en la XIX Feria Internacional del Libro de La Habana, así como

Revista Iberoamericana, Vol. LXXVII, Núm. 235, Abril-Junio 2011, 299-311 
el volumen Soltando amarras y memorias ... de la profesora e investigadora uruguaya Juanamaría Cordones-Cook.

Una breve muestra de sus dibujos, Tra La Lá, es la inaugurada el 30 de abril en el Museo de Marte de la Ciudad de Matanzas a propósito del vigésimo quinto aniversario de Ediciones Vigía.

Revista Iberoamericana, Vol. LXXVII, Núm. 235, Abril-Junio 2011, 299-311
ISSN2154-4794 (Electrónico) 
